American Journal of Pediatrics
2021; 7(3): 178-181
http://www.sciencepublishinggroup.com/j/ajp
doi: 10.11648 /j.ajp.20210703.27
ISSN: $2472-0887$ (Print); ISSN: $2472-0909$ (Online)

\title{
The Value of Calretinin in the Diagnosis of Hirschsprung's Disease in Dakar
}

\author{
Abdou Magib Gaye ${ }^{1, *}$, Gabriel Nougnignon Comlan Deguenonvo ${ }^{2}$, Ibou Thiam ${ }^{1}$, Sophia Raafa $^{3}$, \\ Marie Joseph Dieme-Ahouidi ${ }^{3}$, Cherif Mouhamed Moustapha Dial ${ }^{2}$ \\ ${ }^{1}$ Anatomy and Cytology Laboratory, Aristide Le Dantec Hospital, Dakar, Senegal \\ ${ }^{2}$ Anatomy and Pathological Cytology Laboratory, Idrissa Pouye General Hospital, Dakar, Senegal \\ ${ }^{3}$ Anatomy and Pathological Cytology Laboratory, Fann Hospital, Dakar, Senegal
}

Email address:

gabdomagib@yahoo.fr (A. M. Gaye)

${ }^{*}$ Corresponding author

\section{To cite this article:}

Abdou Magib Gaye, Gabriel Nougnignon Comlan Deguenonvo, Ibou Thiam, Sophia Raafa, Marie Joseph Dieme-Ahouidi, Cherif Mouhamed Moustapha Dial. The Value of Calretinin in the Diagnosis of Hirschsprung's Disease in Dakar. American Journal of Pediatrics. Vol. 7, No. 3, 2021, pp. 178-181. doi: 10.11648/j.ajp.20210703.27

Received: September 3, 2021; Accepted: September 17, 2021; Published: September 26, 2021

\begin{abstract}
Introduction: Hirschsprung's disease is a congenital anomaly characterised by a rarefaction or absence of ganglion cells associated with schwannian hyperplasia or hypertrophy involving the colon or a segment of the colon. Its diagnosis is based on clinical, radiological and histological evidence. Haematoxylin-eosin examination is used in cases of suspected Hirschsprung's disease. However, in some situations it is insufficient to confirm or refute the diagnosis. Immunohistochemical and histo-enzymatic techniques can be used to demonstrate aganglionosis. The aim of our study is to evaluate the interest of calretinin in the diagnosis of Hirschsprung's disease in Dakar. Materials and methods: This was a retrospective and descriptive study from 1 January 2018 to 31 August 2019. It was conducted at the pathology laboratory of Idrissa Pouye General Hospital where 51 paraffin blocks from suspected Hirschsprung's Disease cases were included. All blocks were immunohistochemically studied with anti-calretinin antibody. The diagnosis of Hirschsprung's disease on standard histological examination was based on the absence of lymph node cells in the submucosal and myenteric plexuses. Calretinin immunoreactivity was shown by nuclear and cytoplasmic labelling of ganglion cells and nerve nets. In Hirschsprung's disease, there is an absence of labelling of the nerve plexuses. Results: The majority of patients $(73.5 \%)$ were aged 2 years or older with a mean age of 3.4 years. The sex ratio was 2 . Biopsies constituted $56.86 \%$ of the specimens and surgical specimens $43.14 \%$. Concordance between haematoxylin-eosin examination and calretinin immunohistochemistry was observed in 47 cases $(92.15 \%)$ and discordance in 4 cases $(7.15 \%)$. The sensitivity of calretinin was $93.75 \%$ and the specificity $89.47 \%$. The kappa index was 0.92 . The recto-sigmoid form was the most frequent topographic form observed in $83.3 \%$ of patients. Conclusion: Morphological examination with haematoxylin-eosin remains a good diagnostic method for Hirschsprung's disease. Calretinin immunohistochemistry is necessary in equivocal cases, in neonates and infants and in case of superficial biopsies.
\end{abstract}

Keywords: Hirschsprung, Lymph Node Cells, Calretinin, Dakar

\section{Introduction}

Hirschsprung's disease (HD) is a congenital anomaly of the enteric nervous system responsible for colonic motility disorders usually leading to neonatal intestinal obstruction [1]. The disease affects $1 / 5000$ births, with a sex ratio of 4 boys to 1 girl [2].
In the majority of patients, HD manifests as delayed meconium output in the first few hours of life [3]. It is diagnosed in the neonatal period in $91 \%$ of cases. It can be seen in later life in children, adolescents and exceptionally in adults [4].

The diagnosis of HD is based on clinical and radiological evidence (ASP, barium enema, manometry) but only 
histological examination confirms the diagnosis by demonstrating an absence of lymph node cells associated with schwannian hyperplasia.

Depending on the length of the aganglionic part, $\mathrm{MH}$ is classified as a short variant where the aganglionosis is limited to the sigmoid colon ( $80 \%$ of cases); as a long variant where the aganglionosis reaches or even exceeds the splenic flexure $(17 \%)$, and finally as a total variant which represents $3 \%$ of cases [5].

Proper assessment of the presence of ganglion cells mainly requires adequate sampling and careful tissue preparation. There are many reports on the use of various antibodies in immunohistochemistry (IHC) for better diagnosis of aganglionosis. An immunohistochemical marker will facilitate the detection of ganglion cells through intensive staining, as well as distinguishing ganglion cells from other neuronal components and low artefact rates [6].

Several foreign studies have demonstrated the contribution of calretinin as a marker in the diagnosis of $\mathrm{MH}$ [7].

The objective of our study was to evaluate the value of calretinin in the diagnosis of HD in Dakar.

\section{Materials and Methods}

We collected 51 paraffin blocks in the pathological anatomy laboratory of the Idrissa Pouye General Hospital in Dakar. It concerned 29 biopsies and 22 surgical parts. Thirtytwo (32) blocks concerned the lesion zone and 19 the presumed healthy zone. This work was carried out in collaboration with the pathological anatomy laboratory of the Ibn Rochd hospital in Casablanca (Morocco) where calretinin IHC was performed.

The immuno-histochemical technique was performed in an automated way, Autostainer ThermoFischer $480 \mathrm{~S}$ with positive external controls. The diagnosis of Hirschsprung's disease on standard histological examination was based on the absence of lymph node cells in the submucosal and myenteric plexuses. Calretinin immunoreactivity was shown by nuclear and cytoplasmic labelling of ganglion cells and nerve nets. In Hirschsprung's disease, there is an absence of labelling of the nerve plexuses (figure 1).

The data collected was evaluated and analysed using Excel 2010 and SPSS20.0.

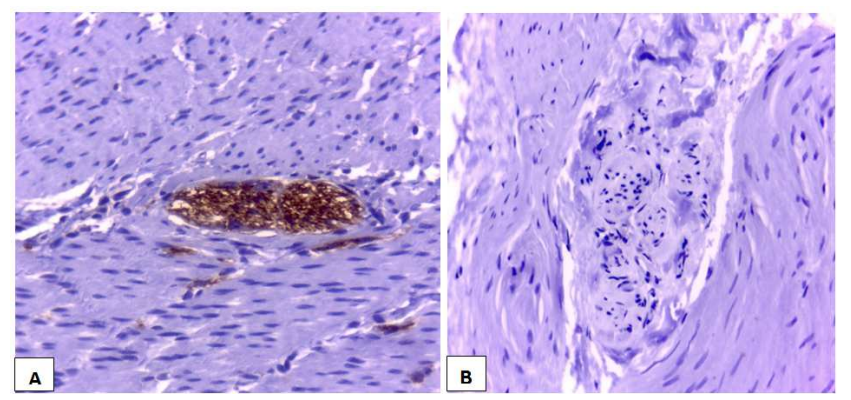

Figure 1. In immunohistochemistry ( $X$ 400), the nerve threads can be cytoplasmic and nuclear labelled with anticalretinin (A). In the case of Hirschsprung's disease, an absence of marking of the nerve nets $(B)$ is noted.

\section{Results}

The mean age of our patients was 3.4 years with extremes of 10 days and 13 years. The age of the patients was greater than or equal to 2 years in $73.5 \%$ of the cases. The predominance was male with 34 boys, i.e. a sex ratio of 2 . Biopsies constituted $56.86 \%$ of the samples and surgical parts $43.14 \%$. The absence of calretinin immunostaining was observed in 30 of the 32 samples taken from the diseased area (aganglion), thus confirming the diagnosis made at haematoxylin- eosin, and calretinin immunoreactivity was noted in 17 of the 19 samples from the presumed healthy zone, i.e. a concordance of $92.15 \%$. A discrepancy was noted in 4 cases ( 2 in the diseased zone and 2 in the presumed healthy zone), i.e. $7.85 \%$. These were two false positives and two false negatives.

The sensitivity of calretinin was $93.75 \%$ and the specificity $89.47 \%$ (kappa index 0.92 ).

The recto-sigmoid form was the most frequent, observed in 14 patients $(83.3 \%)$, the extensive form in 2 patients $(11.8 \%)$ and the total form in one patient $(5.9 \%)$.

\section{Discussion}

The diagnosis of HD is made in most cases in newborns. However, some patients are diagnosed either in childhood or in adulthood [8]. Musa et al [9] studied 48 suspected cases of $\mathrm{HD}$ and found a median age of 1.62 years. Ongeti et al [10] in their study in Kenya, found a median age of 1.94 years. In our study, the mean age of patients was higher due to delayed diagnosis. Indeed, in developing countries, many deliveries are still done at home and in health posts where there is no doctor. In still fragile newborns, nursing is also used after a positive tube test, which delays the biopsy for histological diagnosis. The lack of awareness of HD by some practitioners, or of some of its signs such as abnormal meconium, is also a factor in diagnostic delay.

The male predominance found in our study was reported by other authors. In Ireland, Bradnock [11] studied 304 patients of whom $76.97 \%$ were male.

Granstrom et al [1] reported $78.2 \%$ males in 600 patients with HD in Sweden.

Currently, with the help of IHC, the diagnosis of MH can be made on superficial biopsies (previously considered inadequate with standard techniques) using various markers including calretinin, PS100, NSE, peripherin, synaptophysin, etc [12].

On the OPs, the aim was to confirm the diagnosis, to assess the state of the resection limits and the extent of the lesion.

In our series, biopsy specimens were in the majority with 29 cases, or $56.86 \%$. Kannaiyan L et al [13] and Elhalaby et al [14] reported $60 \%$ and $50.82 \%$ biopsies respectively.

Only histological examination can confirm a definite diagnosis of $\mathrm{MH}$, hence the need to choose the right area to be biopsied to avoid a false positive or false negative result.

Normally, the anal sphincter area contains few or no ganglion cells. 
At the same time, in addition to the lack of ganglion cells in this area, there is hyperplasia of the nerve nets. For this reason, the diagnostic rectal biopsy should be performed well above the pectineal line $(2 \mathrm{~cm}$ in newborns or infants and 3 $\mathrm{cm}$ in children). In addition, biopsies can be difficult to interpret in neonates, as the myenteric plexus cells are often immature at this stage [15]. The pathologist sometimes uses other techniques, such as calretinin IHC to demonstrate the presence or absence of lymph node cells, especially in equivocal cases with absent or scarce lymph node cells without schwannian hyperplasia.

Calretinin is a vitamin D-dependent calcium-binding protein involved in calcium signalling, which plays an important role in the organisation and function of the central nervous system [16].

In our series, a good agreement between the HE result and the IHC result was observed $(92.15 \%)$. This was observed by Keerthy et al [17] in India with a rate of $92 \%$. For Musa et al [9], the concordance was $100 \%$; while it was $88.47 \%$ for Galazka el al [12].

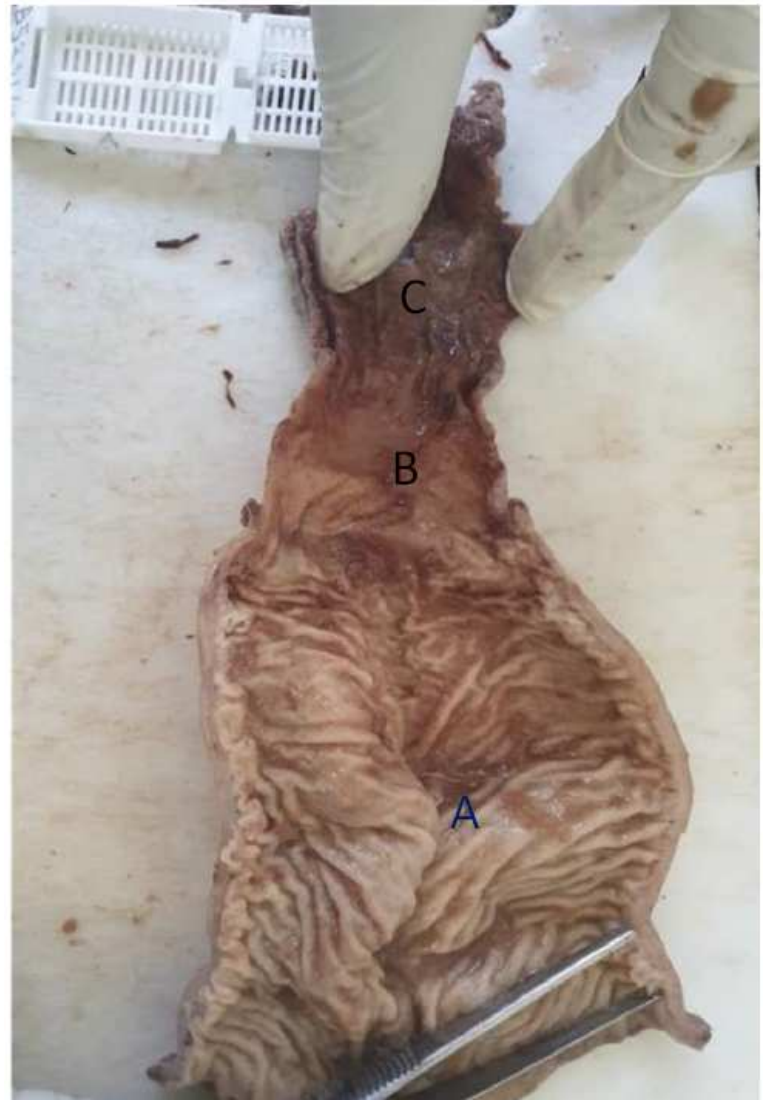

Figure 2. Macroscopic appearance of a colonic resection specimen for Hirschsprung's disease with a dilated healthy zone (A), an intermediate funnel-shaped zone $(B)$ and a narrowed diseased zone $(C)$.

Some authors have investigated the contribution of calretinin IHC examination on samples from the transition zone (figure 2). For example, the study by Rakhshani et al [18] investigated the contribution of calretinin IHC on samples from the transition zone in 94 patients with suspected HCM from 2007 to 2011. Seventy (70) samples were from the lesion zone, 14 from the healthy zone and 10 from the transition zone. A discordant result was observed only on 3 samples from the transition zone where it showed the presence of lymph node cells.

False positives after IHC could be due to labelling of nonnervous cells, mainly mast cells, and more rarely, non-nervous cells that have undergone cytological changes following cytomegalovirus infection. These cells will be mistaken for ganglion cells and rule out a diagnosis of HD [5].

Another hypothesis could be evoked in front of a false positive result. Calretinin-immunoreactive nerve fibres in the lamina propria originating from the colonic ganglion or transition zone may extend into the non-ganglionic part of the colon where the sampling was performed. Nerve net labelling in an aganglionic colonic segment can be a real trap and will require continued evaluation of sufficiently sampled biopsies with adequate submucosa [19].

The good sensitivity and specificity of calretinin in the diagnosis of HD observed in our study $(93.75 \%$ and $89.47 \%$ respectively) was confirmed by Tran et al [20] with a sensitivity of $100 \%$ and a specificity of $99.1 \%$. On the other hand, in the series of Setiadi et al [21] the sensitivity and specificity were $80 \%$ and $75 \%$ respectively.

Regarding the form of $\mathrm{MH}$, the short form was the most frequently observed in the literature. Indeed, in the 2015 cohort of Neuvonen et al [22] in Finland of 146 patients diagnosed with $\mathrm{MH}$, the short form was the most frequent with a rate of $83 \%$, followed by the full form $(10 \%)$, then the extended form (7\%). Bedabrata et al [23] reported in 2016, after studying 104 patients with $\mathrm{MH}$ in India, a predominance of the classic (short) form, found in 83 cases, or $79.8 \%$. Anwarul et al [24] in Bangladesh, in 2018, out of 50 patients, the short form accounted for $72 \%$, the extended form $24 \%$ and the total form $4 \%$.

\section{Conclusion}

Hirschsprung's disease is a congenital anomaly of the enteric nervous system that mainly affects male children aged 2 years and older. The recto-sigmoid form is the most common. Diagnosis is made on rectal biopsy taken $2-3 \mathrm{~cm}$ above the pectineal line. Standard histological examination $(\mathrm{EH})$ is a good method of demonstrating aganglionosis and the concordance is $92.15 \%$ with calretinin IHC. Calretinin IHC is still necessary in equivocal cases, in neonates and infants and in cases of superficial biopsies with only rectal mucosa.

\section{Conflicts of Interest}

The authors declare that they have no competing interests.

\section{Authors' Contributions}

All authors have read and approved the final version of the manuscript. 


\section{References}

[1] Granstrom AL, Svenningsson A, Hagel E. Maternal risk factors and perinatal characteristics for Hirschsprung's disease. Pediatrics 2016; 138: 1508-1512.

[2] Gonzalo DH, Plesec T. Hirschsprung Disease and Use of Calretinin in Inadequate Rectal Suction Biopsies. Arch Pathol Lab Med 2013; 137: 1099-1102.

[3] Meinds RJ, Kuiper GA, Parry K et al. Infant's age influences the accuracy of rectal suction biopsies for diagnosing of Hirschsprung's disease. Clin Gastroenterol Hepatol 2015; 13: 1801-1807.

[4] Singh SJ, Croaker GD, Manglick P et al. Hirschsprung's disease: the Australian Paediatric Surveillance Unit's experience. Pediatr Surg Int 2003; 19: 247-250.

[5] Fernandez RM, Bleda M, Luzon-Toro B et al. Pathways systematically associated to Hirschsprung's disease. Orphanet J Rare Dis 2013; 8: 187.

[6] Kapur RP, Reed RC, Finn LS, Patterson K et al. Calretinin immunohistochemistry versus acetylcholinesterase histochemistry in the evaluation of suction rectal biopsies for Hirschsprung disease. Pediatr Dev Pathol 2009; 12: 6-15.

[7] Hiradfar M, Sharifi N, Khajedaluee M, Zabolinejad N et al. Calretinin immunohistochemistry: an aid in the diagnosis of Hirschsprung's disease.

[8] Hackam DJ, Reblock KK, Redlinger RE, Barksdale Jr EM. Diagnosis and outcome of Hirschsprüng's disease: does age really matter? Pediatr Surg Int 2004; 20 (5): 319-22.

[9] Musa AZ, Quasim BJ, Ghazi HF, Al Chaikhli AWAK. Diagnostic roles of calretinin in hirschprung disease: a comparaison to neuron specific enolase. Saudi J Gastrienterol 2017; 23 (1): 60-66.

[10] Ongeti K, Saidi H, Ogeng'o J, Tharao M. Experience with hirschsprung's Disease at a Tertiary Hospital in Kenya. The Annals of African Surgery 2009, 4: 8-12.

[11] Bradnock TJ, Knight M, Kenny S, Nair M, Walker GM. Hirschsprung's disease in the UK and Ireland: incidence and anomalies. Arch Dis Child 2017; 102: 722-727.

[12] Galazka P, Szylberg L, Bodnar M, Styczynski J et al. Diagnostic Algorithm in Hirschsprung's Disease: Focus on Immunohistochemistry Markers. In vivo 2020; 34: 1355-1359.

[13] Kannaiyan L, Madabhushi S, Malleboyina R, Are NK, Reddy KR, Rao B. Calretinin immunohistochemistry: A new cost- effective and easy method for diagnosis of Hirschsprung's disease. J Indian Assoc Pediatr Surg 2013; 18 (2): 66-68.

[14] Elhalaby ER, Oreiby RM, Hasby AE, Sallam FA. Evaluation of the role of Immunohistochemical markers in the diagnosis of Hirschsprung's Disease. Journal of American Science 2016; 12 (8); 41-50.

[15] Muise ED, Haven RACN. Rectal biopsy for Hirschsprung's disease: A review of techniques, pathology, and complications. World J Pediatr 2016; 12 (2): 135-141.

[16] Guinard-Samuel V, Bonnard A, De Lagausie P, Chomette P, Alberti C, El Ghoneimi A, et al. Calretinin immunohistochemistry: a simple and efficient tool to diagnose Hirschsprung disease. Modern Pathology 2009; 22: 1379 1384.

[17] Keerthi CP, Prema NS. Comparison of Haematoxylin and Eosin Staining and Calretinin Immunohistochemistry in Clinically Suspected Hirschsprung Disease to Evaluate the Diagnostic Utility of Calretinin in Hirschsprung Disease. J. Evolution Med. Dent. Sci 2019; 8 (49): 3702-3706.

[18] Rakhshani N, Araste M, Imanzade F, Panahi M et al. Hirschprung's disease diagnosis: calretinin marker role in determining the presence or absense of ganglion cells. Iran $\mathrm{J}$ Pathol 2016; 11 (4): 409-415.

[19] Hwang S, Kapur RS. Advances abd pitfalls in the diagnosis of Hirschprung's disease. Surgical Pathology 2020: 13 p.

[20] Tran VQ, Lam KT, Truong DQ, Dang MH et al. Diagnostic value of rectal suction using calretinin immunohistochemical staining in Hirschprung's disease. Journal of Pediatric Surgery 2016; 5 p.

[21] Setiadi JA, Dwihantoro A, Iskandar K et al. The utility of hematoxylin and eosin staining in patients with suspected Hirschprung's disease. BMC Surg 2017; 17: 71.

[22] Neuvonen MI, Kyrklund K, Lindahl HG, Koivusalo IA et al. A population-based follow-up of 146 consecutive patients after transanal mucosectomy for Hirschprung's disease. Journal of Pediatric Surgery 2015.

[23] Bedabrata M, Moumita S, Chhanda D, Madhumita M et al. Immunohistochemistry based comparative study in detection of Hirschsprung's disease in infants in a Tertiary Care Center. J Lab Physicians 2017; 9 (2): 76-80.

[24] Anwarul K, Akter M, Tasmiah AT, Hoque M, Tanvir KC, Imam MS et al. Epidemiological caracteristics of Hirschprung's disease; Results of case series of fifty patients of from Bangladesh. Journal of Pediatric Surgery 2018; 53: 1955-1959. 